\title{
Energy intake of Royal Marine recruits relative to training outcomes
}

\author{
T. Davey ${ }^{1}$, S. K. Delves ${ }^{1}$, S. A. Lanham-New ${ }^{2}$, A. J. Allsopp ${ }^{1}$ and J. L. Fallowfield ${ }^{1}$ \\ ${ }^{1}$ Institute of Naval Medicine, Crescent Road, Alverstoke, Gosport, Hants PO12 2DL, UK and ${ }^{2}$ Nutritional Sciences Division, \\ University of Surrey, Guildford, Surrey GU2 7XH, UK
}

The physical demands of Royal Marine (RM) training are such that energy requirements are estimated to be $17572.8 \mathrm{~kJ} . \mathrm{d}^{-1(1)}$ $(1 \mathrm{kcal}=4.184 \mathrm{~kJ})$. It has been hypothesised that a failure to meet this high-energy demand may influence training outcomes. The aim of this study was to examine the effect of energy intake on illness and injury in the Royal Marines; this is part of on-going programme of research being undertaken by the Institute of Naval Medicine examining the dietary intake, nutritional status and bone health of Royal Marines.

RM recruits commencing training at the Commando Training Centre, Royal Marines, Lympstone, Devon were given an initial study brief, after which 545 recruits from eleven Troops consented to participate in the study. Dietary intake was recorded using the Food Record Card (FRC) ${ }^{(2)}$ over $4 \mathrm{~d}$ (including one weekend day) at the start, middle and end of training.

Successful recruits consumed more energy at the middle of training than recruits who failed to complete training $(16936.832 \pm 3966.432 \mathrm{~kJ}$ v. $15334.36 \pm 4589.848 \mathrm{~kJ}(1 \mathrm{kcal}=4.184 \mathrm{~kJ})$, respectively; $P<0.05)$. Recruits reporting a high incidence of illness and/or injury were less likely to complete training. When illness and injury incidence were categorised into quartiles (Table 1), those with the highest illness rates were consuming less energy (as an absolute intake) at the middle of training than those with the lowest illness rates $(P<0.05)$. There was a non-significant trend for a similar pattern with injury incidence $(P=0.08)$. There were no relationships in the proportions of energy intake from carbohydrate, fat or protein relative to illness or injury rates.

Table 1. Daily energy intake relative to illness and injury prevalence

\begin{tabular}{|c|c|c|c|c|c|c|c|c|}
\hline \multirow[b]{3}{*}{ Mid-training intake } & \multicolumn{4}{|c|}{ Illnesses per weeks in training } & \multicolumn{4}{|c|}{ Injuries per weeks in training } \\
\hline & \multicolumn{2}{|c|}{ Lowest quartile ( $n$ 68) } & \multicolumn{2}{|c|}{ Highest quartile ( $n$ 29) } & \multicolumn{2}{|c|}{ Lowest quartile $(n$ 65) } & \multicolumn{2}{|c|}{ Highest quartile ( $n$ 32) } \\
\hline & Mean & SD & Mean & SD & Mean & SD & Mean & SD \\
\hline Energy (kJ) & 17539.32 & 3648.44 & $15819.70 *$ & 4179.82 & 16777.84 & 4481.06 & 15204.66 & 3949.70 \\
\hline Energy $\left(\mathrm{kJ} . \mathrm{kg}^{-1}\right)$ & 953.95 & 200.83 & 866.09 & 251.04 & 907.93 & 234.30 & 815.88 & 225.94 \\
\hline
\end{tabular}

Note: *Significantly different to recruits in the lowest quartile for illnesses $(P<0.05)$.

Energy intake during RM training should be actively encouraged to reduce illness and injury, and promote a successful training outcome. Further research is currently underway to examine the influence of previous lifestyle and nutrition on training outcomes, with particular emphasis on bone health.

The authors would like to thank colleagues at the Commando Training Centre Royal Marines, Lympstone, Devon, for their support and assistance.

1. Alexander DCC, Allsopp AJ \& Pethybridge RJ (1991) Royal Marine Arduous Training Research Project: Nutritional Intake of Royal Marine Recruits. INM Report 10/91.

2. Davey T, Delves SK, Allsopp AJ et al. (2010) Validation of a Bespoke Food Record Card as a Method of Recording Dietary Intake in Royal Marine Recruits. Proc Nutr Soc 2010; 60, 145. 